\title{
Laparoscopic pancreaticoduodenectomy with mesopancreas dissection using artery first approach
}

\author{
Jung Woo LEE*
}

Department of Surgery, Hallym University Medical Center, Seoul, Korea

Introduction: Laparoscopic pancreaticoduodenectomy (LPD) is the one of most technically challenging operations of minimally invasive procedures. Furthermore, LPD remains controversial, especially in the treatment of pancreatic cancer. In this article, the aim is to review our experience of LPD in PDAC that can be performed with safety and good results including oncologic outcome, resection margins and survival rates at a single institution.

Methods: From August 2017 to August 2020, 117 patients underwent LPD in our center. Among 117 patient, 20 patients underwent artery first approach LPD. The patient characteristics, perioperative variables, and post op outcomes were retrospectively collected and analysed.

Results: Seven male and 13 female patients were included in this study. The median age of the patients was 66.8 years. Eleven cases were performed by portal vein resection and reconstruction. Open conversion was none. The average operative time was 475.9 minutes (350-685 minutes). The mean time of superior mesenteric artery clamp was 40.6 minutes (30-55 minutes). Thirty day mortality was not observed in this series. Among 20 patient, 19 patient had negative resection margins, and the median number of lymph node harvested was 17.3 (4-38). The median postoperative hospital stay was 14.3 days (7-24 days). The 1 patient suffered from gastric mucosa bleeding. The 1 patient suffered from grade C POPF.

Conclusions: The artery first approach has been devised to achieve a complete tumor resection for pancreatic cancer. Artery first approach LPD can be safely and feasibly performed in experienced surgeon. However, more study should be required. 